\title{
Acerca de la participación de niños y niñas en actividades de subsistencia Estudio etnográfico en unidades domésticas rurales de Salta (Argentina)
}

\section{Carolina Remorini, ${ }^{1}$ Laura Teves, ${ }^{2}$ María Laura Palermo, ${ }^{3}$ Analía Jacob ${ }^{4}$ y Pilar Desperés}

\author{
1 Universidad Nacional de La Plata. CONICET. Facultad de Ciencias Naturales y Museo. La Plata, Argentina. \\ ORCID iD: 0000-0003-0806-8635 / Correo electrónico: carolina.remorini@gmail.com \\ 2 Universidad Nacional de La Plata. Facultad de Ciencias Naturales y Museo. La Plata, Argentina. \\ ORCID iD: 0000-0003-0806-4271 / Correo electrónico: Iteves@fcnym.unlp.edu.ar \\ 3 Universidad Nacional de La Plata. CONICET. Facultad de Ciencias Naturales y Museo. La Plata, Argentina. \\ ORCID iD: 000o-0002-3036-0352 / Correo electrónico: mlaurapalermo@gmail.com \\ 4 Universidad Nacional de La Plata. Facultad de Ciencias Naturales y Museo. La Plata, Argentina. \\ ORCID iD: 0000-0002-8712-4641 / Correo electrónico: analiajacob@gmail.com \\ 5 Universidad Nacional de La Plata. Facultad de Ciencias Naturales y Museo. La Plata, Argentina. \\ ORCID iD: 0000-0002-6723-3736 / Correo electrónico: pilardesperes@gmail.com
}

Recibido:

28 de diciembre de 2018 Aceptado:

14 de agosto de 2019

doi: 10.34096/runa.v40i2.5503

\section{Resumen}

En este trabajo, analizamos etnográficamente la participación de niños y niñas de comunidades rurales salteñas en actividades de subsistencia doméstica y las habilidades que desarrollan mediante esta participación. A partir de entrevistas a sus cuidadoras, identificamos los modos en que éstas caracterizan comportamientos, disposiciones, habilidades y preferencias de niños y niñas, estableciendo qué tipo de actividades son adecuadas, cómo se asignan, cómo aprenden a llevarlas a cabo y qué cuidados requieren. Asimismo, cómo estas consideraciones pueden favorecer o limitar la participación infantil en la subsistencia. La contribución de niños y niñas es valorada como parte de relaciones de reciprocidad que están en la base de la organización social para tareas productivas, de crianza y cuidado a escala doméstica. Las habilidades que desarrollan durante su participación en la economía doméstica contribuyen a la continuidad de estrategias de vida basadas en el manejo de recursos locales, aun cuando se registran transformaciones a escala local y regional.

Palabras clave

Niñez; Desarrollo; Subsistencia; Unidad doméstica; Salta 


\section{On children's participation in subsistence activities. Ethnogra- phic study in rural households of Salta province (Argentina)}

\begin{abstract}
Key words

Childhood; Development; Subsistence; Domestic unit; Salta

In this paper, we ethnographically analyze the participation of children from rural communities of Salta in domestic activities and the abilities they develop during their participation. Through interviews with their caregivers, we identified the ways in which they characterize children's behaviors, dispositions, abilities, and preferences. Furthermore, it was possible to establish what kind of activities are appropriate, how are they assigned and learned to be carried out by children, and what sort of care they required. Also, we were able to establish how these considerations could stimulate or limit children participation in subsistence activities. Children's contributions are valued as part of the reciprocity relationships that are on the basis of social organization for productive, raising and caring activities at domestic scale. Abilities they develop during their participation in the domestic economy are key to the maintenance of life strategies based on the management of local resources, even when transformations at local and regional scale are registered.
\end{abstract}

\section{Sobre a participação das crianças em atividades de subsis- tência. Estudo etnográfico em unidades domésticas rurais em Salta (Argentina)}

\section{Resumo}

Palavras-chave

Crianças; Desenvolvimento; Subsistência; Unidade doméstica; Salta

\footnotetext{
1. Utilizamos la sigla $\mathrm{NyN}$ tomando como referencia la de NNyA, utilizada en la Encuesta de Actividades de Niños, Niñas y Adolescentes 2016-2017 (INDEC, 2018) para referirse a niños, niñas y adolescentes.
}

Analisamos etnograficamente a participação de crianças de comunidades rurais de Salta em atividades de subsistência domésticas e as habilidades que elas desenvolvem por meio dessa participação. Através de entrevistas com suas cuidadoras identificamos os modos pelos quais eles caracterizam comportamentos, disposições, habilidades e preferências das crianças, estabelecendo quais atividades são apropriadas, como elas são outorgadas, como eles aprendem a realizá-las e quais os cuidados necessários. Do mesmo modo, como essas considerações podem favorecer ou limitar a participação infantil na subsistência. A contribuição das crianças é valorizada como parte das relações recíprocas que estão na base da organização social para tarefas produtivas, de criação e cuidado em escala doméstica. As habilidades que as crianças desenvolvem durante sua participação na economia doméstica contribuem para a continuidade de estratégias de vida baseadas na gestão de recursos locais, ainda quando há transformações registradas nas escalas locais e regionais.

\section{Introducción}

La participación de niños y niñas (en adelante, $\mathrm{NyN})^{1}$ en actividades rutinarias que contribuyen a la subsistencia de sus grupos domésticos ha sido extensamente documentada por investigaciones etnográficas y transculturales (Gaskins, 2000, 2006; Morelli, Rogoff y Angelillo, 2003; Rogoff, Paradise, MejíaArauz, Correa-Chávez y Angelillo, 2003; Bolin, 2006; Invernizzi, 2007; Ramírez Sánchez, 2007; Rogoff et al., 2007; Weisner y Gallimore 2008; Klein, Graesch e Izquierdo, 2009; Rogoff, Morelli y Chavajay, 2010; Ames, 2013; Alcalá, Rogoff, Mejía-Arauz, Coppens y Dexter, 2014; Coppens, Alcalá, Mejía-Arauz y Rogoff, 
2014). Éstas muestran que existe un amplio rango de tareas que contribuyen a la subsistencia, que van desde el lavado de utensilios, la colaboración en la obtención y preparación de alimentos, la elaboración de productos para la venta, hasta el cuidado de hermanos/as menores. Esta última, por ejemplo, facilita las tareas productivas de los adultos/as a la vez que constituye una instancia en que $\mathrm{NyN}$ mayores enseñan o introducen a los/as menores en tareas de valor para la subsistencia.

Tal compromiso con las actividades comunitarias es un componente central del desarrollo infantil, ya que su participación en las rutinas domésticas es el contexto por excelencia en el cual desarrollan habilidades para devenir sujetos competentes (Weisner, 1984; Gauvain, 1998; Rogoff, 2003, 2010). La relevancia de las actividades en las que $\mathrm{NyN}$ son incluidos -o a las que se atrae su atención-es destacada por Urie Bronfenbrenner (1987), ya que son los "hechos ambientales" con mayor impacto en el desarrollo (Bronfenbrenner, 1987; Weisner, 1998; Ingold, 2000; Rogoff, 2003; Remorini, 2015). Según Bronfenbrenner (1987), la ecología del desarrollo humano considera el desarrollo como resultante de la participación activa y cambiante en diversas actividades y rutinas cotidianas, y son estas las que definen qué habilidades son "ecológicamente relevantes" (Ingold, 2000; Greenfield, Heller, Fuligni y Mayrand, 2003).

Los adultos/as les asignan tareas y responsabilidades según valores y expectativas acerca de sus habilidades y madurez en cada etapa de la niñez; junto con la evaluación de sus disposiciones, actitudes y preferencias (Remorini, 2009). En muchas comunidades, los adultos/as favorecen que NyN tomen la iniciativa para llevar a cabo determinadas tareas, ya que lo consideran indispensable para su aprendizaje (Bolin, 2006; Remorini, 2009, 2013; Ames, 2013; Alcalá et al., 2014; Coppens et al., 2014; Rogoff, 2014). La posibilidad de que $\mathrm{NyN}$ se comprometan en tareas domésticas está directamente asociada con oportunidades en las cuales la composición de la unidad doméstica, los patrones de organización social de las actividades y las representaciones de los cuidadores/as sobre el desarrollo infantil juegan un rol central (Lancy, 2016).

En numerosas circunstancias, las tareas realizadas por $\mathrm{NyN}$ suelen ser reconocidas por los adultos/as como "entrenamiento" o "ayuda", especialmente cuando se las asocia a situaciones lúdicas, en las que ensayan y ponen en práctica habilidades mientras juegan (Padawer y Enriz, 2009; Remorini, 2009; Padawer 2015). Sin embargo, esta visión de las actividades infantiles únicamente como preparación para la vida adulta oscurece la comprensión de la agencia infantil, la cual permite considerarlos como sujetos que tienen un rol y una participación en diferentes dominios de sus vidas, según su etapa vital (Uprichard, 2008; Remorini, 2015; Gallois y Reyes García, 2018).

En este trabajo caracterizamos las maneras en que $\mathrm{NyN}$ de diferentes edades de comunidades rurales de los Valles Calchaquíes (Salta) participan en actividades que contribuyen a la subsistencia doméstica y las habilidades que desarrollan mediante esta participación desde la perspectiva de sus cuidadoras (madres y abuelas). Nuestro análisis parte de conceptos como "comunidad de práctica" (Lave y Wenger, 1991) y "participación intensa en comunidades de práctica" (Rogoff et al., 2003), que suponen el intercambio, aprendizaje mutuo, coordinación y cooperación entre "aprendices" y "expertos/as". Dependiendo de la actividad, "aprendices" y "expertos/as" tienen roles específicos, interactúan de diversas maneras en función de la edad, sus relaciones de parentesco y posición en el grupo doméstico (Padawer, 2015). Sobre esta base, nos proponemos identificar las representaciones de las cuidadoras sobre el aprendizaje 
de habilidades relevantes para el desarrollo de $\mathrm{NyN}$, y cómo estas se traducen en comportamientos y disposiciones que pueden favorecer o limitar su participación en actividades de subsistencia. Nos enfocamos en las tareas que ellos/as llevan a cabo, los criterios para asignarlas y la organización social y espacial que favorece tal participación. Nos interesa enfatizar que su contribución a la subsistencia es valorada como parte de las relaciones de reciprocidad que están en la base de la organización social para las tareas productivas, de crianza y cuidado. $\mathrm{NyN}$ son integrantes plenos de sus grupos domésticos, y les son reconocidos derechos y obligaciones como tales, por lo tanto, están fuertemente comprometidos con estas tareas. Hacia el final, discutimos el papel de estas habilidades ecológicamente relevantes para el aprendizaje en contextos domésticos intergeneracionales y productivos.

\section{Acerca de Molinos}

Este artículo surge de una investigación etnográfica en curso en el departamento de Molinos (Salta), centrada en el análisis de la crianza y cuidado de la salud a escala doméstica y su impacto en las trayectorias de desarrollo infantil. Molinos se localiza en la porción meridional de los Valles Calchaquíes salteños, y su población asciende a 5652 habitantes ( 2785 hombres y 2867 mujeres) de los cuales unos 3500 residen en las zonas rurales, con un índice de masculinidad menor al 95\% (Instituto Nacional de Estadística y Censos [INDEC], 2010).

La localidad cabecera es referida como "el pueblo" (o "el bajo"), y las áreas rurales son denominadas "fincas" (los "cerros" o el "alto"). La fundación del pueblo data de mediados del siglo XVII y actualmente cuenta con 1166 habitantes según censo local del 2015. Las fincas son propiedades privadas dedicadas a la producción agrícola-ganadera y artesanal; combinan sistemas tradicionales de utilización de mano de obra (arriendo, mediería y pastaje) con la producción para la subsistencia para consumo de la unidad

2. Estos territorios son propiedad de familias de hacendados, legado que puede rastrearse desde la conquista española a la actualidad. El modelo de trabajo de la tierra y el usufructo de la producción tiene sus orígenes en la encomienda española. Cada familia que prestaba servicio a la finca permanecía de por vida sujeta a esta relación, y adquiría incluso compromisos laborales para las generaciones siguientes, las de sus hijos y nietos. En las últimas décadas, las familias de campesinos o puesteros cambiaron su relación de trabajo con los finqueros por medio de acuerdos para el arriendo de la tierra, el usufructo compartido de la producción o el pago por el pastaje para la cría de animales. Durante la década de 1990, algunas de las fincas familiares fueron vendidas y adquiridas por capitales extranjeros. Sin embargo, los finqueros mantienen el control de recursos en los territorios de sus propiedades, lo que incide fundamentalmente en el acceso al agua de regadío y en el control de la mano de obra local involucrada en los trabajos de producción (Teves, 2011). doméstica. $^{2}$

La reconstrucción de los procesos que han atravesado la vida de las poblaciones de los Valles Calchaquíes desde la existencia de los grupos originarios, las influencias de otros pueblos andinos, la conquista española y el surgimiento de las poblaciones mestizas hasta el presente dan cuenta de visiones contrapuestas sobre los modos de apropiación del territorio y de explotación de los recursos (Teves, 2002). La influencia de diversos patrones culturales y los procesos de mestizaje tornan necesaria la consideración de los movimientos de poblaciones, resistencia y conflictos, así como los repoblamientos desde tiempos arqueológicos e históricos hasta la actualidad (Göbel, 2000).

No obstante las transformaciones ocurridas como resultado de estos procesos, la economía de los valles se basa fuertemente en la utilización de recursos locales, la producción y consumo a escala doméstica, articulada con diferentes modalidades productivas y comerciales a escala local y regional. Esta economía se funda en una especial relación material y simbólica entre las comunidades y su entorno (Göbel, 2000), a través de actividades y modos de organización que garantizan la preservación de la biodiversidad y la continuidad de prácticas culturales asociadas a su manejo.

Parte de la población vallista se radica en el pueblo, donde la presencia de campos para el cultivo y/o la cría de animales es limitada. No obstante, mantiene lazos de parentesco y cooperación con pobladores de las fincas, lo que les 
permite acceder a recursos localizados en el "alto", al combinar un modo de subsistencia tradicional con otro basado en ingresos provenientes de empleos formales e informales y programas sociales. Quienes residen en las fincas practican tareas agrícola-ganaderas para el autoconsumo y para la finca, y también reciben ingresos de programas sociales. Estos dos modos de asentamiento no son excluyentes, ya que algunos de los habitantes poseen propiedades en el pueblo y en las fincas, lo que permite desarrollar un esquema de permanencia alternada entre estos enclaves, así como alojar transitoriamente parientes y amigos que puedan seguir este patrón de desplazamiento (Morgante y Remorini, 2018).

La producción agrícola consiste en el cultivo de maíz, pimiento, alfalfa, cebolla, vid, frutales y aromáticas. El consumo doméstico y el intercambio es la prioridad de las familias de los sectores altos y bajos del valle. La cría de ganado se vincula asimismo con la producción artesanal de tejidos la cual representa una fuente de ingresos importante. La cría se hace a escala familiar y de modo extensivo, lo que desplaza los rebaños de vacas, cabras, ovejas y llamas por extensas zonas de pastos naturales, en zonas cercanas a las viviendas o en "el alto". Esta actividad se complementa con la obtención de subproductos como leche, quesos y charqui (Teves, 2011).

Recientemente, numerosas fincas con bodegas artesanales han sido adquiridas por industriales extranjeros y argentinos, como consecuencia de lo cual se registra un auge de la actividad vitivinícola y del turismo asociado a ella. El avance de esta modalidad productiva conlleva una notable disminución de la población en las fincas y, en algunos casos, la relocalización de la población en el pueblo u otros lugares de la región del NOA.

\section{Metodología}

Este trabajo se basa en una estrategia metodológica de tipo cualitativo, adecuada a investigaciones que se orientan a formular inductivamente preguntas e hipótesis, a partir de los datos relevados en un contexto específico. En este sentido, la perspectiva y metodología etnográfica resultan productivas para dar cuenta de los conocimientos, expectativas y valores de las cuidadoras sobre el aprendizaje de habilidades relevantes para el desarrollo de NyN, así como de los contextos que dan sentido a dichos conocimientos y valores.

Los datos en los que se basa este artículo fueron obtenidos en trabajos de campo realizados en 2013, 2017 y 2018, en unidades domésticas (UD) localizadas en la zona rural, en un valle de altura, denominado aquí "el cerro". ${ }^{3} \mathrm{La}$ selección de UD se basó en la presencia de $\mathrm{NyN}$ menores de diferentes edades, y en la diversidad en su tamaño y composición según género y edad.

En el marco de nuestra investigación y en tres relevamientos empíricos realizamos entrevistas semiestructuradas a cuidadoras que habitan en el cerro, todas ellas mujeres, quienes fueron entrevistadas en más de una oportunidad. Las entrevistas se orientaron principalmente a caracterizar: representaciones acerca de la salud y el bienestar de $\mathrm{NyN}$ y de los riesgos en diferentes momentos de la vida; sus formas de prevención, diagnóstico y tratamiento; representaciones, expectativas y valores en torno al desarrollo de $\mathrm{NyN}$ y sus competencias para participar en diferentes actividades y entornos; fundamentos de las prácticas de crianza y cuidado registradas; criterios, recursos y vínculos sociales que se ponen en juego en el desarrollo de las actividades cotidianas de crianza y
3. A los fines de preservar la identidad de los pobladores del área bajo estudio, se ha decidido evitar utilizar los nombres de localidades, instituciones y personas. Esta investigación fue sometida a evaluación y aprobada desde el punto de vista ético por un comité de ética independiente de Salta. Todos los entrevistados prestaron su consentimiento libre e informado y, de acuerdo con la ley 25.326, sus datos personales, así como los de los niños y niñas participantes del estudio han sido protegidos. 
cuidado de los NyN; expectativas y valoración de la conducta y actividades cotidianas de los NyN y su contribución a la subsistencia de la UD. Para cada UD, relevamos genealogías y datos sociodemográficos, y complementamos esta información con registros de instituciones de salud.

Junto con las entrevistas realizamos observaciones (spot, continuas durante varias horas del día y participante) en cuatro UD, que enfocaron en: comportamientos y actividades de $\mathrm{NyN}$ en el espacio doméstico (alimentación, movilidad, exploración, juego, descanso, tareas relacionadas con la subsistencia); interacciones entre $\mathrm{NyN}$ y cuidadores/as-otros adultos/as-otros niños/ as durante actividades de crianza y cuidado; actividades de los cuidadores (adultos/as y/o niños/as) en relación directa con NyN; actitudes y disposiciones que favorecen/limitan la participación de $\mathrm{NyN}$ en tareas de subsistencia; formas de enseñar o demostrar a NyN.

Registramos la información en audio, grillas y diarios de campo, y complementamos este registro con fotografías y video.

A los fines de este artículo, seleccionamos 12 entrevistas realizadas a nueve cuidadoras de entre 33 y 42 años de cuatro UD, las que varían en cuanto a tamaño, la presencia de hombres adultos, el tipo de organización familiar (nuclear/extensa) y la relación con el trabajo asalariado para la finca. Si bien esta selección no pretende ser representativa en términos estadísticos, reúne un conjunto que casos que dan cuenta de aspectos comunes y diferenciales de modos de organización doméstica rural y su incidencia en la participación de $\mathrm{NyN}$ en la economía doméstica. Asimismo, complementamos esta información con aquella proveniente de observaciones de las rutinas cotidianas, registrada mediante notas de campo, que ofrece información contextual de interés para enmarcar la narrativa de las entrevistadas.

En cuanto al análisis, se utilizó el software Nvivo 11, para formalizar, codificar y sistematizar los datos cualitativos e identificar categorías locales y temas emergentes (nodos) para la descripción del campo conceptual y sus relaciones jerárquicas. Algunos de los interrogantes que guiaron nuestro análisis fueron: ¿de qué modo $\mathrm{NyN}$ se ven involucrados en las actividades domésticas, incluyendo tareas en el hogar, o aquellas relacionadas con la recolección, agricultura y/o cría de ganado?; ¿cómo interactúan con otros sujetos durante el desarrollo de tales actividades?; ¿cómo identifican las cuidadoras diversas habilidades que les permiten a $\mathrm{NyN}$ formar parte de diferentes espacios y actividades de subsistencia?; ¿de qué manera esto se traduce en prácticas que promueven o limitan la contribución infantil a estas actividades?; ¿qué expectativas y valores expresan las cuidadoras acerca de la contribución de $\mathrm{NyN}$ a la subsistencia de la unidad doméstica?

Focalizamos en la narrativa acerca del modo en que las cuidadoras caracterizan comportamientos, actitudes, disposiciones, habilidades y preferencias de $\mathrm{NyN}$. Partimos de considerar que tales descripciones son consistentes con valores y expectativas cultural e históricamente construidas, que se transforman con el tiempo, varían intra e intergeneracionalmente y se fundan en diferentes fuentes de conocimiento con grado diverso de legitimación (Remorini, 2015). Siguiendo a Murphy (2007), nos interesa analizar cómo el discurso "conforma" o "construye" NyN "normales" y/o "competentes", al establecer qué tipo de actividades son adecuadas a ellos/as, cómo aprenden a llevarlas a cabo, qué cuidados requieren y qué "peligros" pueden comprometer su bienestar durante su desarrollo. En tal sentido, analizamos cómo tales discursos ponen 
de relieve ideas, supuestos y valores que se comparten y actualizan intergeneracionalmente en diferentes ámbitos de la vida cotidiana.

\section{Acerca de las unidades domésticas de las fincas}

Partimos de considerar que las unidades domésticas de las áreas rurales constituyen la base de la producción, consumo y sociabilidad local. Definimos la UD como la unidad compleja que incluye un componente social -grupo de personas que comparten la residencia- y un componente espacial -el espacio físico que habitan-, articulados por un conjunto de actividades relevantes a la subsistencia del grupo que se realizan parcial o totalmente en ese ámbito (Crivos y Martínez, 1996).

Esta noción delimita un espacio social y simbólico en el que se despliegan las interacciones entre las personas que lo integran. Existe un fuerte arraigo local, dado por la pertenencia a "la familia" y al "valle". A pesar de la autonomía relativa de las UD en cuanto a la producción y el manejo de los recursos locales, estas mantienen vínculos parentales, de reciprocidad y de cooperación con individuos y "familias" que se extienden más allá de la finca, y que se manifiestan en la realización de actividades de subsistencia y rituales (por ejemplo: señalada, primer corte de cabello -ruti-, fiestas patronales). Estas relaciones también se ponen de manifiesto en circunstancias que requieren de la ayuda mutua o el apoyo social o material para determinados problemas (por ejemplo, cuidado de la salud, escolaridad de los hijos/as).

Las actividades de subsistencia incluyen todas aquellas que permiten a los miembros de una UD contribuir a su reproducción -entendida esta como un proceso multidimensional que involucra aspectos biológicos, materiales y sociales (Eguía y Ortale 2004)-, desde la producción de alimentos hasta el cuidado de sus integrantes (Daltabuit Godás, 1992). En la mayoría de las familias campesinas, la participación de las mujeres, $\mathrm{NyN}$ en la economía resulta crucial "casi todas las obligaciones hogareñas de las mujeres contribuyen a la producción y reproducción de la fuerza de trabajo [...] no sólo criando niños sino también con su cuidado y socialización [...]" (Daltabuit Godás, 1992, p. 18).

El modo de organización doméstica vallista garantiza la convivencia de, al menos, tres generaciones, unidas básicamente por parentesco consanguíneo o afinal. Tradicionalmente, las UD siguen un patrón matrifocal, y en algunos casos presentan generaciones alternas. Considerando la ausencia temporaria o definitiva de jóvenes y adultos/as, resulta una forma de organización recurrente en la cual los más viejos se encargan parcial o completamente de la crianza de los nietos/as (Morgante y Remorini, 2018).

Las rutinas de las UD con presencia estable de hombres adultos son diferentes de aquellas que incluyen mujeres y sus hijos/as y nietos/as en cuanto a la distribución del tiempo, la asignación de tareas entre adultos, $\mathrm{NyN}$ y el involucramiento de los hombres en la crianza de NyN. Los hombres adultos comparten pocas horas al día durante la semana con el resto de su familia, la interacción es más frecuente los fines de semana o cuando ellos no están afectados a las labores asalariadas dentro o fuera de la finca. En estas circunstancias, se espera la contribución de niños, niñas y jóvenes a diversas actividades de subsistencia, acorde con la evaluación que hacen los adultos/ as de sus posibilidades y habilidades. 
La vida cotidiana de los integrantes de estas UD se desarrolla básicamente dentro de los límites de la "casa", conformada esta por una compleja articulación de espacios abiertos y habitaciones con funciones específicas y que, en conjunto, alcanzan amplias dimensiones. Los espacios se delimitan de acuerdo con sus usos productivos (uno o más corrales según tipos de animales, chacras, rastrojos, cocinas de "verano" e "invierno" con sus respectivas kanchas para fogones, horno de barro, morteros, u otros), como habitación (dormitorios), como lugar de reunión familiar (galerías semiabiertas) o como lugares de almacenaje de productos o bienes personales. Cada UD puede tener, además, uno o más "puestos" en el cerro.

$\mathrm{NyN}$ y adultos/as de diferentes edades comparten los espacios de producción y reunión, en relación con lo cual se observa una integración intergeneracional en las actividades rutinarias. Son principalmente las mujeres, $\mathrm{NyN}$ quienes diariamente se ocupan del ganado, y los hombres, de la agricultura para el consumo doméstico o para la finca. Ellos también intervienen en la construcción de corrales y en otras tareas que exigen mayor esfuerzo o experticia (marca, castración, por ejemplo). Si no hay hombres en la UD, se puede "conchabar" a hombres de otras familias.

Asimismo, algunas UD están conformadas por miembros que no se dedican a la cría de ganado, sino que trabajan de forma asalariada en la finca. En estos casos, venden sus animales o los entregan a otras familias "arrendándolos" o dándolos "al partir". Los vínculos entre UD se ponen de manifiesto asimismo en actividades que requieren de la participación conjunta y cooperación, por ejemplo, durante las "corridas" de ganado que se realizan anualmente. Estas actividades, así como muchas otras, generan contextos en los que participan miembros de distintas generaciones, que conforman "comunidades de práctica" (Lave y Wenger, 1991) y contribuyen a la reproducción de la UD.

\section{La contribución de los niños a la subsistencia de la unidad doméstica}

En esta sección, focalizamos en la narrativa de las cuidadoras en torno a la participación de $\mathrm{NyN}$ en actividades de subsistencia. Al respecto, es preciso subrayar que nos referimos principalmente a $\mathrm{NyN}$ cuyas edades oscilan entre los 5 y 12 años, ya que son, por un lado, los que intervienen más frecuente y activamente en estas actividades, y por otro, quienes permanecen en la UD la mayor parte del tiempo, puesto que cuando inician la educación secundaria deben trasladarse al pueblo y permanecer allí durante la semana.

Los $\mathrm{NyN}$ más pequeños son denominados guaguas, término que deriva del quechua wawá y que, si bien se utiliza de modo general para designar a NyN de pecho antes de que comiencen a caminar (De Suremain y Montibert, 2007), algunas personas lo aplican también para referir al feto y a $\mathrm{NyN}$ hasta la edad escolar; es el único término indígena que se conserva para referir a las etapas en el curso vital (Remorini, 2013). A diferencia de las guaguas, que son "tiernitos" (vulnerables) y dependientes - por lo que hay que destinar mayor esfuerzo a su cuidado-, los NyN a partir de los 4-5 años poseen mayor autonomía, son capaces de colaborar y/o asumir responsabilidades domésticas y están expuestos a un rango menor de "peligros" que pueden comprometer su desarrollo y bienestar (Remorini y Palermo, 2016). Estos/as suelen ser denominados "jardineros" o "escoleros", lo que pone de relieve que una actividad central a su vida cotidiana es la asistencia a las instituciones escolares. En el 
caso de algunas UD, NyN permanecen albergados de lunes a viernes en la escuela, debido a que la distancia entre esta y sus viviendas impide el traslado diario. Ello conlleva que la participación de $\mathrm{NyN}$ en las actividades de estas UD se restrinja a los fines de semana y recesos escolares.

Como hemos señalado en otro lugar (Remorini, 2013), a medida que crecen, los $\mathrm{NyN}$ pequeños/as son integrados al grupo de hermanos y hermanas y cuidados por ellos/as -lo que permite a los adultos/as continuar con sus actividades-, con lo cual tempranamente aprenden a ajustar su conducta a las rutinas domésticas. En este sentido, se espera que los $\mathrm{NyN}$ con cierto grado de autonomía se adecuen a las rutinas del hogar y no al revés. Según las cuidadoras, deben aprender a ser "responsables", a no ser "vagos", y a combinar el juego con actividades "útiles" que les permitan aprender lo necesario para su vida adulta. En síntesis, su autonomía, junto con la obediencia y la responsabilidad, son actitudes altamente valoradas.

Identificamos en esta narrativa un conjunto de expectativas acerca de la contribución de $\mathrm{NyN}$ a las actividades de subsistencia y la valoración de sus habilidades que les permiten llevar a cabo tales tareas en colaboración con otros/ as. Tomamos de Ingold (2000) el concepto de habilidad, la cual es construida e incorporada por medio de la práctica y el entrenamiento en un compromiso íntimo con el ambiente; no viene dada por una determinación genética o por la interiorización de representaciones colectivas. Es la capacidad de acción y percepción del organismo situado en un ambiente ricamente estructurado. Las habilidades son reconstruidas en cada generación, incorporadas al modus operandi del organismo en desarrollo a partir del entrenamiento y la experiencia en la realización de una actividad particular. Es decir que el proceso de "habilitación" es indisociable de los contextos prácticos de actividad en los que se produce tal capacitación.

El proceso por el cual NyN desarrollan habilidades "ecológicamente relevantes" para la vida en los cerros está favorecido por la disposición de las cuidadoras a reconocer su iniciativa y decisión de integrarse a tareas que les son de interés sin que los adultos/as ordenen o coaccionen. Son "expuestos" a entornos y actividades gracias a la integración social y espacial (Greenfield et al., 2003; Rogoff, 2003; Lancy, 2016) que prima en las UD de los cerros, y los adultos y otros niños y niñas juegan un rol central en educar u orientar su atención hacia actividades y eventos en el ambiente doméstico que son relevantes (Remorini, 2015).

En todos los relatos, las cuidadoras subrayan su intención de dar lugar a la iniciativa de $\mathrm{NyN}$, siempre que la tarea sea acorde con sus posibilidades y no represente un riesgo evidente.

Ellos cuando le saltan ganas se lo das a hacer. [...] Se le ocurre hacer cualquier cosa, por ahí el machete, se van a hachar ramas. Pero por ahí yo los tengo cortitos porque es un peligro que se pueden hachar, se pueden cortar. Van a juntar leña, o a ayudarme en algo aquí. (299, Salta, febrero 2017)

De todo hacen. Yo voy a buscar leña y ellas van a traer leña... Cabras que están cerca, las mando a traer, traen. Sacan la leche, hacen queso. Hacen la masa también. Una, el otro día me ha dicho que ella hace la masa para cenar, para hacer las tortillas. Y ha hecho, yo la he ayudado, y ha hecho la masa. (272, Salta, febrero 2017) 
Las cuidadoras plantean asimismo que en la decisión de asignar o no una tarea a sus hijos/as, deben contemplar que muchas veces estos las realizan mientras "juegan": "Si la mando a M con G se ponen a jugar y se quedan hasta cualquier hora. Los mando a la mañana, se quedan hasta el mediodía. Tengo que llamarlos. Entonces, muy pocas veces los mando juntos" (340, Salta, febrero 2017).

También reconocen preferencias y disposiciones particulares, lo que conforma un criterio para la distribución de las tareas: “El N, flojo es. 'Ya lavo, ya lavo' y nunca lava. Nunca lava... Le gusta más el cerro, ver las cabras. Le mandamos a traer las cabras y va" (272, Salta, febrero 2017).

En cuanto a las actividades del hogar en las que NyN de los cerros "colaboran", las más frecuentes son: recolectar leña, acarrear agua, lavar su ropa, colaborar en la preparación de alimentos, "hacer queso" y lavar "el servicio" (platos y cubiertos luego de la comida). También son ellos/as los encargados de hacer un "mandado", es decir, de ir a pedir o llevar algún objeto de una vivienda a otra o transmitir algún mensaje.

De los que están conmigo, sea que uno va a largar las vacas, otro va a largar las ovejas, otro cocina, otro lava los servicios, otro barre. De todo hacen ellos [...] de chiquitas, ya así de la edad de los changuitos, así, ya ellas juntaban leña, ya, no lo hacían tan bien, pero ya ellas ya traían; [ahora] arman los quesos, cuando no estaba yo, ellas ya ordeñaban, traían las cabras, sacaban leche. (299, Salta, mayo 2018)

Las tareas de recolección y acarreo de leña y agua requieren de habilidades específicas y, a su vez, están atravesadas por situaciones que pueden convertirse en potenciales riesgos. Con relación a la leña, implica poder identificar no solo las especies adecuadas como combustible, sino los espacios donde se localizan y la cantidad necesaria. Este conocimiento de los recursos y los espacios se desarrollan en tanto los niños desde pequeños "acompañan", aunque, hasta que no desarrollan suficiente fuerza física para acarrear leña, no toman parte de la actividad como los mayores. “De chiquitos van con nosotros a juntar leña, ya de cuatro años, así. Ya van, andan con nosotros. No juntan leña, pero andan" (272, Salta, febrero 2017).

En estas instancias, $\mathrm{NyN}$ recolectan además plantas medicinales o alimenticias, lo que pone de relieve sus conocimientos sobre nombres de especies, distribución espacial y propiedades (Zarger, 2002; Martínez y Pochettino, 2004; Miller, 2006; Setalaphruk y Price, 2007; Morelli, 2012). Asimismo, nuestra observación de estas actividades muestra las diferentes estrategias que ellos/as ponen en juego para recolectar y transportar estos recursos, lo que da cuenta de habilidades cognitivas complejas, como cálculo de distancias y cantidades, utilización y/o transformación de herramientas, junto con las motrices implicadas en el desplazamiento y transporte.

Esta instancia de "acompañamiento" es, desde la perspectiva de las cuidadoras, un contexto óptimo para el aprendizaje inicial de la actividad, mediante la observación, la escucha atenta y la sincronización con los otros que forman parte de ella. El discurso resalta el carácter progresivo del aprendizaje y pone de relieve que no es necesario que $\mathrm{NyN}$ dominen ciertas habilidades para tomar parte de la actividad.

De todo lo que hacemos, de a poquito, ya sé que no lo harán bien [ahora], que no lo harán todo, pero tienen que ir aprendiendo desde chiquitos. Porque si no, grandes 
pues, ellos van a ser los que van a sufrir si nosotros no le dejamos hacer nada. (272, Salta, febrero, 2017)

Los llevo a palear, a por ahí con palas, por ahí. No les mezquino yo, que se vayan porque así van a aprender. (299, Salta, mayo 2018)

En cuanto a las actividades agrícola-ganaderas, $\mathrm{NyN}$ participan-mayormente junto con otros sujetos- en las tareas de riego, armando cercos para los animales, "paleando" y "macheteando", "semillando" (plantar) "deshierbando" (desmalezar), ordeñando, "sacando" y "cuidando" (vigilando) la "hacienda" (ganado); acompañando a los animales mientras comen, "trayendo" la hacienda (reunir y llevar los animales al corral luego de soltarlas para alimentarse), e integrando el grupo que sale para "las corridas", circunstancias en las se les asignan diferentes tareas acordes con sus habilidades.

A ya tiene 8 años. Él ya ayuda a tirar del caballo, ayuda a tirar las semillitas, él sabe cuánto, le vamos diciendo, de a cinco papitas, así, en esa distancia, o el maíz más lejitos... [la más chiquita] todavía no.... Quizás ya este año puede... ella saca yuyitos, separa, pero semillar no, porque si va y tropieza y ya se cae todo (las semillas). (227, Salta, febrero 2013)

También la asignación varía en función de la distribución de tareas según la composición de la UD.

Con las cabras son un día la M [su tía], ella va a buscar las cabras con sus chicos. Y otro día voy yo con los chicos [...] (¿Vos lavás la ropa de todos tus nenes?) de los cinco más chicos, ya $\mathrm{S}$ [15 años] a veces se lava su ropa, la lava él los sábados ya... Y cuando tenemos que regar o barrer tierra, o algunas cosas, ahí sí, él hace [...] Cuando no hace nada, ya se pone a lavar su ropa; a veces le mando a buscar al burro, para buscar la mercadería aquí, yo ya le lavo ahí. (272, Salta, febrero 2017)

Cuando la UD está integrada solo por personas "viejas" ("abuelitos/as"), lo que en las fincas suele ocurrir cuando los más jóvenes migran al pueblo o la ciudad, los padres suelen atender la solicitud de estos de contar con la ayuda temporaria de un nieto/a, elemento clave en la reciprocidad entre viejos/as, jóvenes y niños/as en este valle. "Porque ahora ella se ha enfermado (su abuela) y después no tenía que hacer fuerza ni nada. Él (su hijo) ya está para ayudar, juntar leña, para el agua, y para ver las cabras también... lo que necesite" (340, Salta, febrero 2017).

El manejo del agua para riego es una actividad en la que NyN "ayudan" bajo la orientación de los/as mayores: asisten "abriendo" o "trancando" (cerrando) la circulación del agua por acequias y canales dentro de los arriendos, utilizando rocas $u$ otros elementos, en una tarea coordinada con otros que garantiza el cumplimiento de la actividad en el tiempo adecuado, es decir, optimizando el recurso el día que su familia tiene "turno" para regar. Si por alguna razón los adultos/as de la UD no pueden asumir esa tarea, no pueden asignar esta responsabilidad a los NyN, y deben pedir ayuda a otra UD o "conchabar un peón".

Las tareas vinculadas a la cría y manejo de animales requieren de un alto grado de coordinación y sincronización entre los participantes, y del reconocimiento de la contribución que cada uno/a puede hacer al logro de la actividad. En ocasiones, los adultos describen la participación de NyN como "ayuda", y 
4. Las corridas de ganado se reconocen como una actividad propia de los habitantes de las zonas rurales. Se realizan durante los meses de mayo o junio de cada año y consisten en el arreo del ganado vacuno desde los cerros hasta las zonas de quebrada o corrales. El objetivo es correr a los animales dispersos, agruparlos y movilizarlos hacia otros sectores, marcarlos y vacunarlos. La corrida implica una organización entre vecinos y parientes que colaboran durante un periodo de diez días, aproximadamente (Jakel y Teves, 2015). distinguen sus intervenciones de aquellas que requieren mayor experticia y responsabilidad.

[las más grandes] algunas veces que tienen tiempo nos ayudan, y si no ellas están a tiro con su estudio. Como la chica grande ya es colegiala, así que ya tiene que estar ahí estudiando. Generalmente sábado después doce y domingo. Días de semana no tenemos ayuda. (299, Salta, mayo 2018)

Las cuidadoras destacan el contacto íntimo de algunos NyN con "su haciendita", y reconocen que solo algunos/as "se interesan" y anticipan una dedicación a la cría de animales cuando sean adultos, mientras que otros tempranamente muestran una orientación y dedicación mayor hacia el estudio, que los lleva a invertir más tiempo en la escuela y proyectar una trayectoria educativa fuera de la finca. "Cuando tienen mucha aflicción, ya solitos dicen 'me voy a ver la hacienda' 'me voy a ver las cabras' 'me voy a ver las ovejas'”' (299, Salta, febrero 2017).

La mayor oportunidad que tienen los $\mathrm{NyN}$ de los cerros para integrarse a las actividades de cría de ganado se relaciona fundamentalmente con la adquisición de animales desde el nacimiento y en diferentes instancias de la trayectoria vital. En la infancia, NyN pueden obtener ganado "propio" mediante regalos e intercambios, en instancias como el bautismo o el ruti. Si bien en la infancia y niñez se delega la responsabilidad en los adultos/as, $\mathrm{NyN}$ colaboran en diferentes tareas, como el cuidado, la señalada y la marcada.

Durante las "corridas", 4 hombres y mujeres coordinan un cronograma de salidas y disponen todo lo relativo a la logística para la captura de animales. Los hombres salen caminando, a caballo y con camionetas hacia las zonas altas o de pastaje del ganado. Las mujeres, $\mathrm{NyN}$ también intervienen en esta actividad; en general, llegan caminando o con mulas hasta los sectores donde se improvisan los campamentos. Ellas llevan las provisiones y los elementos para preparar las comidas de los hombres. NyN también participan en las corridas y colaboran en actividades consideradas "menores", como acarreo de agua y leña, o en la preparación de la comida. Aquí también los más grandes muestran sus habilidades con el lazo y "ayudan" dentro de los corrales (Jakel y Teves, 2015) con la aplicación de vacunas y en la "marcada". Cuando las familias bajan de los cerros, al finalizar esta etapa, se organizan "carneadas" en las que NyN solamente observan o asisten a los adultos trayendo utensilios, pero no intervienen directamente en la tarea.

Las cuidadoras destacan la afinidad que tienen $\mathrm{NyN}$ con algunos animales a los que "mezquinan" o por los que sienten "aflicción" (preocupación) y, en consecuencia, prestan especial atención. Este fuerte compromiso emocional con los animales se manifiesta ante la pérdida o disminución del ganado, lo que NyN y adultos/as vivencian como sufrir de "pena".

NyN son capaces de reconocer, desde muy pequeños, cuáles son "sus" animales por características físicas y comportamentales, y en virtud de estas, les colocan nombres específicos. También, saben cuántos animales tiene cada uno, e intervienen en las decisiones sobre su cuidado, trueque y/o venta. Cada UD tiene sus rebaños; algunos de ellos alcanzan los cientos de animales, y los niños/ as heredan algunos de sus parientes; luego, su rebaño se incrementa mediante el "multiplico" si es que tienen "suerte". En ocasiones, pueden decidir cambiarlos por dinero para solventar sus estudios en el pueblo, o para comprar bienes como ropa, motos o celulares. 
NyN se involucran en tareas ganaderas ordinarias y extraordinarias, con una participación que va desde el acompañamiento, al inicio, a una intervención en secuencias de tareas específicas, por sí solos o en colaboración con otros individuos. Ya a los 13-14 años se considera que tanto varones como mujeres pueden hacerse cargo de su hacienda. Para que NyN sean parte de estas actividades y puedan sostenerlas en el tiempo, deben reunir asimismo, además del interés, condiciones personales, algunas de las cuales suelen reunirse bajo la categoría "suerte".

La experiencia cercana de $\mathrm{NyN}$ con el ganado posibilita el desarrollo de habilidades motrices como montar a caballo/jinetear, lo que requiere de un control del propio cuerpo y del cuerpo del animal; también aprender a armar lazos y enlazar, a rodear a los animales. Asimismo, supone desarrollar habilidades cognitivas y sensoriales (atención, escucha, observación e identificación de atributos de los animales, reconocimiento de riesgos, conocimiento de espacios y recursos naturales). Por último, habilidades emocionales vinculadas con la empatía que se requiere para el cuidado, el reconocimiento de perspectivas e intereses de otros participantes, necesarias para la coordinación y sincronización durante la actividad (Bolin, 2006; Ng'asike, 2010).

La cría de ganado implica asimismo un patrón de movilidad espacial y social que impacta en el conocimiento que adquieren $\mathrm{NyN}$ acerca del manejo del espacio y la circulación por caminos, corrales y refugios que pertenecen a "sus familias" y que también comparten con otras. En la trayectoria personal de numerosas mujeres y hombres se advierte la importancia de esta actividad, en tanto la hacienda constituye una base económica que respalda otras estrategias de subsistencia doméstica. La cría del ganado supone una dedicación completa de tiempo, en particular durante algunos meses del año, pero en algunos casos también puede conjugarse con otras oportunidades de trabajo. Algunos jóvenes, por ejemplo, optan por tomar trabajos asalariados en la ciudad, pero se comprometen a regresar en las temporadas de captura, marcada y señalada de animales, para ayudar a sus parientes.

La continuidad en el tiempo en el manejo del ganado parece justificar la trasmisión de una herencia económica y simbólica, pero también la transferencia de un conjunto de habilidades complejas y vínculos sociales, los que en su mayoría son mantenidos y compartidos intergeneracionalmente con los miembros de otras UD localizadas en la región o fuera de ella.

\section{Consideraciones finales}

Los resultados presentados aquí dan cuenta de aspectos poco explorados hasta el momento en las comunidades rurales de los Valles Calchaquíes Salteños, ya que en general los estudios se han centrado en las actividades y saberes de los adultos y "expertos" en determinadas actividades y/o tecnologías (Crivos, 2004; Teves, 2011). Este trabajo constituye un primer acercamiento sistemático al tema, basado en fuentes verbales, que necesitará ser profundizado mediante la incorporación de datos observacionales. Aunque, de modo preliminar, permite caracterizar la contribución de los conocimientos y actividades infantiles a la economía doméstica, y por extensión, a la continuidad de un modo de vida basado en un tipo de relación con el entorno que favorece la preservación de la diversidad ecológica de la región andina. 
El enfoque adoptado permite dialogar con otras investigaciones en contextos ecológicos y culturales diversos centrados en la contribución infantil a la subsistencia doméstica, con énfasis en los procesos de aprendizaje implicados y en el valor de las habilidades desarrolladas, no sólo para la ejecución de las actividades en cuestión sino para su transferencia a otros dominios de la vida comunitaria (Rogoff, 2014).

En este sentido, la participación infantil en las rutinas domésticas es considerada clave en varios planos. Por un lado, porque $\mathrm{NyN}$ no solo aprenden conocimientos y habilidades técnicas, sino también modos de relación e interacción, y valores culturales como la reciprocidad entre generaciones (Ramírez Sánchez, 2007). Por otro lado, porque aporta concretamente a la reproducción de las UD mediante tareas específicas que asumen mayormente las mujeres y niños/as. Esta contribución es destacada por las investigaciones que focalizan en la economía rural, la que actualmente combina la producción para el autoconsumo con diferentes modalidades de intercambio, comercio y trabajo asalariado, dentro y fuera del sector rural (Daltabuit Godás, 1992; Padawer, 2015; Gaskins, 2006; Padawer y Enriz, 2009; Tassinari, 2012).

Nuestro estudio a escala etnográfica del proceso de desarrollo de habilidades de NyN plantea la necesidad de articular en futuras instancias los datos discursivos con aquellos que resultan de la observación de tales contextos de actividad. Ello nos permitirá identificar y recuperar las lógicas subyacentes a las formas de participación y cooperación, centradas en la observación atenta, minuciosa y precisa, la atención focalizada, la imitación y la escucha con expectativas de involucrarse en la actividad, como preludio ineludible para otras formas de intervención y dominio técnico de habilidades (Rogoff, 2014). En este sentido, posibilitará reconocer variables para caracterizar la dinámica social implicada en estos procesos de aprendizaje y evaluar su posible transferencia a la descripción de otros contextos comunitarios.

Podemos presuponer que el carácter cognitivo y práctico de estos procesos es dinámico y que requiere ser minuciosamente explorado. Este trabajo muestra cómo las cuidadoras relatan las actividades de subsistencia en las que participan $\mathrm{NyN}$ y muestran aspectos y acciones que, desde sus puntos de vista, facilitan el desarrollo gradual de las habilidades infantiles. Esto nos permite, como antropólogos, delimitar campos de observación para el registro de datos sobre cómo $\mathrm{NyN}$ son habilitados en estas comunidades; y al mismo tiempo, nos conduce a la obtención de información que integra y articula estas conductas con niveles de organización familiar, comunitario y económico más amplios.

Recuperar la minuciosidad de la descripción etnográfica para esclarecer las vinculaciones entre actores y niveles contextuales hace posible jerarquizar el protagonismo de $\mathrm{NyN}$ en la reproducción de la UD, en contraposición con formulaciones que fueron duramente criticadas ante la pérdida de visibilidad de estos actores. Asimismo, esta descripción permite discutir los límites entre las actividades "productivas" y otras que no se consideran como tales y que remiten a dimensiones económicas, simbólicas y sociales a la vez (Trinchero, Balazote y Valverde, 2007), como el caso de la cría de ganado explorado en este trabajo.

La combinación de actividades de subsistencia doméstica local y las actividades productivas a escala regional y de valor en el mercado plantean un nuevo escenario para el desarrollo de habilidades, no solo de aquellas que se adquieren en el ámbito doméstico sino de las que se desarrollan en el ámbito 
educativo. No estamos caracterizando las UD como unidades cerradas, sino por el contrario, como permeables e insertas en contextos diversos y frente a experiencias de interacción con otros conceptos de economía, como la de los grupos empresariales locales y extranjeros. En este sentido, los cultivos y el ganado tienen valor cultural y económico en tanto potencialmente puedan conectar la vida cotidiana de los pobladores de altura con otros escenarios que demandan habilidades "tradicionales" y nuevas para la subsistencia de estas comunidades. Un ejemplo de ello es el conocimiento que tienen $\mathrm{NyN}$ acerca del valor cultural de las corridas de ganado y de la cooperación entre familias para su cuidado, pero a temprana edad saben también acerca del valor como capital y moneda que tiene su ganado y su carácter de bien personal, por el cual ellos solos pueden disponer de su venta.

Las actividades descritas en este trabajo parecen asociarse únicamente a aquellas consideradas "tradicionales" en los grupos campesinos andinos. Sin embargo, esperamos que las observaciones y registros en contextos extradomésticos permitan identificar nuevos intereses de los cuidadores por introducir a $\mathrm{NyN}$ en actividades que acompañan cambios en la subsistencia, en sus relaciones con nuevos sectores y demandas a diferentes niveles. Finalmente, es esperable que el enfoque etnográfico facilite la identificación de variables teórico-metodológicas que contribuyan a la modelización de las conductas de NyN y a la explicación de su dinámica en el ámbito doméstico y comunitario más amplio. Y que estas variables sirvan a efectos de una análisis comparativo que dé cuenta de las dimensiones "horizontales", o entre dominios cognitivos, y "verticales", es decir, que articulen los contextos micro, meso y marco de análisis de las prácticas de subsistencia actuales.

\section{Financiamiento:}

Este documento es resultado del financiamiento otorgado por el Estado Nacional (CONICET y UNLP), por lo tanto queda sujeto al cumplimiento de la Ley № 26.899.

Los datos presentados aquí derivan del Proyecto 11/N821 financiado por la Universidad Nacional de La Plata "Etnografía y redes sociales aplicadas al estudio de las estrategias de manejo del medioambiente en dos comunidades de Argentina", dirigido por Laura Teves y Carolina Remorini; de la investigación aprobada y financiada por el Consejo Nacional de Investigaciones Científicas y Técnicas (CONICET) a Carolina Remorini como miembro de la carrera de investigador científico; así como de la investigación enmarcada en la beca doctoral de María Laura Palermo, financiada por el mismo organismo. Asimismo, del Proyecto de Investigación Plurianual 0270 del Consejo Nacional de Investigaciones Científicas y Técnicas (CONICET) denominado: "Trayectorias, actividades y redes sociales emergentes: Productividad del uso de 'métodos mixtos' en la caracterización de las estrategias de vida en la región del NOA".

\section{Agradecimientos:}

Deseamos agradecer a las mujeres entrevistadas, por su calidez y hospitalidad, por su interés y colaboración en nuestra investigación. Asimismo, a las autoridades municipales, eclesiásticas y a directivos y personal de Molinos, por su permanente colaboración y facilitación de condiciones para la realización de nuestro trabajo de campo. 


\section{Sobre las autoras}

Carolina Remorini es Investigadora adjunta CONICET. Profesora titular en la asignatura Etnografía I (FCNyM-UNLP). Integrante del Laboratorio de Investigaciones en Etnografía Aplicada (LINEA-FCNyM-UNLP). La Plata, Argentina.

Laura Teves es Investigadora de la UNLP. Profesora adjunta en la asignatura Orientaciones en la Teoría Antropológica. Integrante del Laboratorio de Investigaciones en Etnografía Aplicada (LINEA-FCNyM-UNLP). La Plata, Argentina.

María Laura Palermo es Becaria doctoral del CONICET. Ayudante diplomada en la asignatura Etnografía I (FCNyM-UNLP). Integrante del Laboratorio de Investigaciones en Etnografía Aplicada (LINEA-FCNyM-UNLP). La Plata, Argentina.

Analía Jacob es Becaria doctoral UNLP. Ayudante de segunda interina en la asignatura Antropología Sociocultural II (FCNyM-UNLP). Integrante del Laboratorio de Investigaciones en Etnografía Aplicada (LINEA-FCNyM-UNLP). La Plata, Argentina.

Pilar Desperés es Becaria CIN-UNLP. Auxiliar alumna ad-honorem a través del programa de adscripción a la docencia en la asignatura Antropología Social y Cultural (FPs-UNLP). Integrante del Laboratorio de Investigaciones en Etnografía Aplicada (LINEA-FCNyMUNLP). La Plata, Argentina. 


\section{Q Referencias bibliográficas}

» Alcalá, L., Rogoff, B., Mejía-Arauz, R., Coppens, y Dexter, A. L. (2014). Children's Initiative in Contributions to Family Work in Indigenous-Heritage and Cosmopolitan Communities in Mexico. Human Development, 57, 96-115.

»Ames, P. (2013). Niños y niñas andinos en el Perú: crecer en un mundo de relaciones y responsabilidades. Boletín del Instituto Francés de Estudios Andinos (BIFEA), 42(3), 389409.

"Bolin, I. (2006). Growing up in a Culture of Respect. Child Rearing in Highland Peru. Austin, TX: The University of Texas Press.

» Bronfenbrenner, U. (1987). La ecología del desarrollo humano: Experimentos en entornos naturales y diseñados. Barcelona: Paidós.

" Coppens, A., Alcalá, L., Mejía-Arauz, R. y Rogoff, B. (2014). Children's Initiative in Family Household Work in Mexico. Human Development, 57, 116-130. doi: 10.1159/000356768

" Crivos, M. (2004). Contribución al estudio antropológico de la medicina tradicional de los Valles Calchaquíes (Provincia de Salta). (Tesis de Doctorado). La Plata: Universidad Nacional de La Plata.

»Crivos, M. y Martínez, M. R. (1996). Las estrategias frente a la enfermedad en Molinos (Salta, Argentina). Una propuesta para el relevamiento de información empírica en el dominio de la etnobiología. En Contribuciones a la Antropología Física Latinoamericana (pp. 99-104). Memoria del IV Simposio de Antropología Física “Luis Montané”. Instituto de Investigaciones Antropológicas UNAM/Museo Antropológico Luis Montané, Universidad de La Habana.

"Daltabuit Godás, M. (1992). Mujeres mayas: Trabajo, nutrición y fecundidad. Ciudad de México: Universidad Autónoma de México.

»De Suremain, C.-É. y Montibert, N. (2007). Au fil de la faja. Enrouler et dérouler la vie en Bolivie. En D. Bonnet y L. Pourchez (Eds.), Du soin au rite dans l'enfance (85-102). París: Érès.

»Eguía, A. C. y Ortale, M. S. (2004). Reproducción social y pobreza urbana. Cuestiones de Sociología, 2, 21-49.

» Gallois, S. y Reyes-García, V. (2018). Children and Ethnobiology. Journal of Ethnobiology, 38(2), 155-169. Recuperado de https://doi.org/10.2993/0278-0771-38.2.155

»Gaskins, S. (2000). Children's Daily Activities in a Mayan Village: A Culturally Grounded Description. Cross-Cultural Research, 34(4), 375-389. https://doi. org/10.1177/106939710003400405

"Gaskins, S. (2006). The Cultural Organization of Yucatec Mayan Children's Social Interactions. En X. Chen, D. C. French y Schneider (Eds.), Peer Relationships in Cultural Context. (Cambridge Studies in Social and Emotional Development, pp. 283-309). Cambridge: Cambridge University Press. doi:10.1017/CBO9780511499739.013

» Gauvain, M. (1998). Cognitive development in social and cultural context. Current Directions in Psychological Sciences, 7, 188-192.

» Göbel, B. (2000). Identidades sociales y medio ambiente: la multiplicidad de los significados del espacio en la puna de Atacama. Cuadernos del Instituto Nacional de Antropología y Pensamiento Latinoamericano, 19, 267-296. 
» Greenfield, P., Heller, K., Fuligni, A. y Mayrand, A. (2003). Cultural Pathways Through Universal Development. Anual Review of Psychology, 54, 461-49o.

» Instituto Nacional de Estadística y Censos (INDEC) (2010). Censo Nacional Argentino 2010. Recuperado de http://www.indec.gov.ar/censos_provinciales2010

» INDEC (2018). Encuesta de Actividades de Niños, Niñas y Adolescentes 2016-2017. Ciudad Autónoma de Buenos Aires: Instituto Nacional de Estadística y Censos-INDEC.

» Ingold, T. (2000). The Perception of the Environment: Essays on livelihood, dwelling and skill. Londres y Nueva York: Routledge.

» Invernizzi, A. (2007). Children's Work as Participation. Thoughts on Ethnographic Data in Lima and the Algarve. En B. Hungerland, M. Liebel, B. Milne y A. Wihstutz (Eds.), Working to be Someone. Children Focused Research and Practice with Working Children. (pp. 135-144). London: Jessica Kingsley.

» Jakel, A. y Teves, L. (2015) Las corridas de ganado en molinos: una propuesta de etnografía visual sobre la trashumancia de ganado en los Valles Calchaquíes septentrionales, Salta, Argentina. Iluminuras, 16(40), 85-132. Recuperado de http://seer.ufrgs. br/index. php/iluminuras/article/view/61243

»Klein, W., Graesch, A. e Izquierdo, C. (2009). Children and Chores: A Mixed-Methods Study of Children's Household Work in Los Angeles Families. Anthropology of Work Review, 98-109. doi:10.1111/j.1548-1417.2009.01030

» Lancy, D. 2016. Playing with Knives: The Socialization of Self-Initiated Learners. Child Development, 87(3), 654-665. doi: 10.1111/cdev.12498

» Lave, J. y Wenger, E. (1991). Situated learning. Legitimate peripheral participation. Cambridge: Cambridge University Press.

» Martínez, M. R. y Pochettino, M. L. (2004). Microambientes y recursos vegetales terapéuticos. Conocimiento local en Molinos, Salta, Argentina. Zonas Áridas, 8 (1), pp.18-31. http://dx.doi.org/10.21704/za.v8i1.708

» Miller, D. (2006). The Seeds of Learning: Young Children Develop Important Skills through their Gardening Activities at a Midwestern Early Education Program. Applied Environmental Education y Communication, 6(1), 49-66. doi: 10.108o/15330150701318828

» Morelli, C. (2012). Teaching in the Rainforest: Exploring Matses Children's Affective Engagement and Multisensory Experiences in the Classroom Environment. Teaching Anthropology, 2(2), 53-65.

» Morelli, G., Rogoff, B. y Angelillo, C. (2003). Cultural variation in young children's access to work or involvement in specialised child-focused activities. International Journal of Behavioral Development, 27, 264-274.

» Morgante, M. G. y Remorini, C. (2018). Estudio etnográfico de las relaciones intergeneracionales en el cuidado de la salud a escala doméstica durante las etapas pre y postnatal (Molinos, Salta, Argentina). Apuntes, Revista de Ciencias Sociales, 45(83), 37-65.

» Murphy, E. (2007). Images of childhood in mothers' accounts of contemporary childrearing. Childhood, 14(1), 105-127.

» Ng'asike, J. T. (2010). Turkana Children's Sociocultural Practices of Pastoralist Lifestyles and Science Curriculum and Instruction in Kenyan Early Childhood Education (tesis de doctorado). Tempe, AZ: Arizona State University.

» Padawer, A. (2015). Infancia y trabajo a través de las generaciones: la transmisión de conocimientos vinculados a la reproducción social en contextos rurales en transformación. Revista de Ciências Sociais Política y Trabalho, 43, 113-132. 
»Padawer, A. y Enriz, N. (2009). Experiencias formativas en la infancia rural mbyá-guaraní. Avá. Revista de Antropología, 15, 315-332.

»Ramírez Sánchez, M. A. (2007). “Helping at Home”: The Concept of Childhood and Work among the Nahuas of Tlaxcala, México. En B. Hungerland, M. Liebel, B. Milne y A. Wihstutz (Eds.), Working to be Someone. Children Focused Research and Practice with Working Children. (pp. 87-97). London: Jessica Kingsley.

» Remorini, C. (2009). Aporte a la Caracterización Etnográfica de los Procesos de SaludEnfermedad en las Primeras Etapas del Ciclo Vital, en Comunidades Mbya-Guaraní de Misiones, República Argentina (tesis de doctorado). Facultad de Ciencias Naturales y Museo. Universidad Nacional de La Plata. La Plata: EDULP.

» Remorini, C. (2013). Estudio etnográfico de la crianza y la participación infantil en comunidades rurales de los Valles Calchaquíes Septentrionales, Noroeste Argentino. Resultados preliminares. Boletín del Instituto Francés de Estudios Andinos (BIFEA), 42(3), 411-433.

» Remorini, C. (2015). Learning to Inhabit the Forest: Autonomy and Interdependence of Lives from a Mbya-Guarani Perspective. Advances in Child Development and Behavior. Special issue on Children Learn by Observing and Contributing to Family and Community Endeavors A Cultural Paradigm, 49, pp. 273-288. Eds: M. Correa-Chávez, R. Mejía-Arauz y B. Rogoff. doi:10.1016/bs.acdb.2015.09.003

» Remorini, C. y Palermo, M. L. (2016). “Los míos... itodos asustados!”: vulnerabilidad infantil y trayectorias de desarrollo en los Valles Calchaquíes Salteños. Mitológicas, XXXI, 83-112.

» Rogoff, B. (2003). The cultural nature of human development. Oxford: Oxford University Press.

» Rogoff, B. (2014). Learning by Observing and Pitching In to Family and Community Endeavors: An Orientation. Human Development, 57, 69-81.

» Rogoff, B., Paradise, R., Mejía Arauz, R., Correa-Chávez, M. y Angelillo, C. (2003). Firsthand learning through intent participation. Annual Review of Psychology, 54(1), 175-203.

» Rogoff B., Moore, L., Behnosh, N., Dexter, A., Correa-Chávez, M. y Solís, J. (2007). Children's Development of Cultural Repertoires Through Participation in Everyday Routines and Practices En J. E. Grusec y P. D. Hastings (Eds.), Handbook of Socialization (pp. 490-515). Nueva York: Guilford.

» Rogoff, B., Morelli, G. A. y Chavajay, P. (2010). Children's Integration in Communities and Segregation From People of Differing Ages. Perspectives on Psychological Science, 5(4), 431-440. Recuperado de http://pps.sagepub.com/content/5/4/431

»Setalaphruk, C. y Price, L. (2007). Children's traditional ecological knowledge of wild food resources: A case study in a rural village in Northeast Thailand. Journal of Ethnobiology and Ethnomedicine, 3:33. https://doi.org/10.1186/1746-4269-3-33

" Tassinari, A. (2012). A participação de crianças agricultoras na produção de alimentos. Simposio Children's Food Heritage. Anthropological Issues. $54^{\circ}$ Congreso Internacional de Americanistas, 15-20 de julio. Viena.

» Teves, L. (2002). Trazas de paisajes en Molinos, un pueblo del Valle Calchaquí, Salta, Argentina. En P. Denis y J. Worthington. The Power of Oral History: Memory, Healing and Development, Ph. Pietermaritzburg. vol. 1. (p. 145-155). Sudáfrica: International Oral History Association y Universidad de Natal.

» Teves, L. (2011). El Estudio Etnográfico de la Actividad Textil como aporte a la Caracterización del Modo de Vida en el Pueblo de Molinos y zona de influencia (Provincia de Salta). (Tesis de doctorado). Universidad Nacional de La Plata, Facultad de Ciencias Naturales y Museo, La Plata, Argentina. 
» Trinchero, H., Balazote, A. y Valverde, S. (2007). Antropología económica y ecológica: recorridos y desafíos disciplinares. Cuadernos de Antropología Social, 26, 7-19.

» Uprichard, E. (2008). Children as "being and becomings": children, childhood, and temporality. Children \& Society, 22, 303-313.

"Weisner, T. (1984). Ecocultural niches of middle childhood: A cross-cultural perspective. En A. Collins (Ed.), Development during middle childhood (pp. 335-369). Washington, DC: National Academy Press.

"Weisner, T. (1998). Human development, child well-being and the cultural project of development. New Directions for Child Development 1998 (80), 69-85. https://doi. org/10.1002/cd.23219988006

» Weisner, T. S., y Gallimore, R. (2008). Child and sibling caregiving. En R. S. New (Ed.), Anthropology and child development: A cross-cultural reader (pp. 264-269). Malden: Blackwell.

» Zarger, R. (2002). Children's Ethnoecological Knowledge: Situated Learning and the Cultural Transmission of Subsistence Knowledge and Skills among Q'eqchi' Maya (PhD Dissertation). EEUU: The University of Georgia. Recuperado de https://getd.libs.uga.edu/pdfs/zarger_rebecca_k_200208_phd.pdf 